\title{
MicroRNA-133b inhibits connective tissue growth factor in colorectal cancer and correlates with the clinical stage of the disease
}

\author{
YIHANG GUO $^{1}$, XIAORONG LI ${ }^{1}$, CHANGWEI LIN ${ }^{1}$, YI ZHANG ${ }^{2}$, GUI HU $^{1}$, \\ JIANYU ZHOU ${ }^{1}$, JUAN DU ${ }^{1}$, KAI GAO ${ }^{1}$, YI GAN ${ }^{1}$ and $\mathrm{HAO} \mathrm{DENG}^{3}$ \\ ${ }^{1}$ Department of General Surgery, The Third Xiangya Hospital of Central South University; \\ ${ }^{2}$ Department of General Surgery, The Xiangya Hospital of Central South University; ${ }^{3}$ Center for Experimental Medicine, \\ The Third Xiangya Hospital of Central South University, Changsha, Hunan 410013, P.R. China
}

Received February 19, 2014; Accepted November 14, 2014

DOI: $10.3892 / \mathrm{mmr} .2014 .3075$

\begin{abstract}
Accumulating evidence indicates that dysregulation of microRNA-133b (miR-133b) is an important step in the development of certain types of human cancer and contributes to tumorigenesis. Altered expression of miR-133b has been reported in colon carcinoma, but its association with clinical stage in colorectal cancer (CRC) has remained elusive. Connective tissue growth factor (CTGF), a potentially promising candidate gene for interaction with miR-133b, was screened using microarray analysis. The expression of miR-133b and CTGF was evaluated using reverse transcription-quantitative polymerase chain reaction and western blot analysis. The regulatory effects of miR-133b on CTGF were evaluated using a dual-luciferase reporter assay. CTGF was identified as a functional target of miR-133b. The results demonstrated low expression of miR-133b in CRC specimens with poor cell differentiation $(\mathrm{P}=0.011)$, lymph node metastasis $(\mathrm{P}=0.037)$ and advanced clinical stages (stage III or IV vs. I or II; $\mathrm{P}=0.036)$. Furthermore, there was a significant association between a high level of expression of CTGF mRNA and an advanced clinical stage (stage III or IV vs. I or II; $\mathrm{P}=0.015)$ and lymph node metastasis $(\mathrm{P}=0.034)$. CTGF expression was negatively regulated by miR-133b in the human colorectum, suggesting that miR-133b and CTGF may be candidate therapeutic targets in colorectal cancer.
\end{abstract}

\section{Introduction}

Colorectal cancer (CRC) is the most common malignancy of the digestive tract worldwide (1), and the incidence is steadily

Correspondence to: Professor Xiaorong Li, Department of General Surgery, The Third XiangYa Hospital of Central South University, 138 Tongzipo Road, Changsha, Hunan 410013, P.R. China

E-mail: lixiaorong@medmail.com.cn

Key words: microRNA-133b, connective tissue growth factor, colorectal cancer, tumor nodes metastasis stage increasing, partly as a result of recent changes in lifestyle and dietary habits (2). Survival rates in CRC depend on early diagnosis and treatment. However, the lack of a reliable biomarkers of CRC remains a significant obstacle to a timely diagnosis.

Recent studies have suggested that dysregulation of microRNAs (miRNAs) is an important step in the development of a number of types of cancer, including CRC. miRNAs may function as potent regulators of gene expression and altered miRNA levels may result in the aberrant expression of gene products that are involved in tumorigenesis (3). miR-133b was initially hypothesized to be a muscle-enriched miRNA. Through targeting of genes involved in cardiac development and cardiac channel expression, miR-133b has been implicated in the regulation of cardiac myogenesis and cardiac ion channel expression (4). It has recently been reported that the expression of miR-133b is downregulated in gastric and esophageal adenocarcinomas as well as colorectal tumors $(5,6)$. However, its role and relevance in colorectal cancer remains largely unknown.

Connective tissue growth factor (CTGF) is a member of the $\mathrm{CCN}$ cysteine-rich family of proteins. These proteins are known to stimulate mitosis, adhesion, apoptosis, extracellular matrix production, growth arrest and migration in numerous cell types (7). CTGF is known to be important in the prevention of hypoxia-induced apoptosis and the promotion of epithelial-mesenchymal transistion (EMT) in tumor-reactive stroma; in addition, CTGF has been shown to be involved in the development of malignancy in a number of organs (8). A series of studies have suggested that a high level of CTGF expression is associated with tumor progression and clinical stage (9-14). CTGF expression is induced by transforming growth factor (TGF) $\beta$ as well as other prohypertrophic stimuli, such as endothelin. In addition, studies have reported that the regulation of CTGF expression may be correlated with that of miR-145 and miR-30 (4,15). Of note, a bioinformatics targetscan analysis indicated putative miR-133b binding sites within the 3'-untranslated region (UTR) of CTGF.

In the present study, the expression pattern of miR-133b and CTGF was examined in human CRC tissues and CRC cell lines. In addition, the possible target genes of miR-133b were identified. Furthermore, the association between the 
expression of miR-133b and CTGF and various clinicopathological parameters was investigated.

\section{Materials and methods}

Cell culture and reagents. Human HT-29 and SW-620 CRC cell lines were obtained from the Cell Center of Xiangya School of Medicine, Central South University (Hunan, China). The human CCD-18Co healthy colon cell line was obtained from the American Type Culture Collection (Manassas, VA, USA). RPMI-1640 was obtained from Invitrogen Life Technologies (Carlsbad, CA, USA). Fetal bovine serum (FBS) was purchased from Sijiqing Biological Engineering Materials Co., Ltd. (Hangzhou, China). Cells were cultured at $37^{\circ} \mathrm{C}$ in a humidified incubator with 5\% $\mathrm{CO}_{2}$ and maintained in RPMI-1640 supplemented with heat-inactivated 10\% FBS (Invitrogen, Carlsbad, CA, USA), $100 \mathrm{U} / \mathrm{ml}$ penicillin and $100 \mu \mathrm{g} / \mathrm{ml}$ streptomycin (Sijiqing Biological Engineering Materials Co., Ltd). Serum-free Opti-MEM ${ }^{\circledR}$ medium was obtained from Invitrogen Life Technologies. Protease inhibitor was obtained from AppliChem (Gatersleben, Germany). miR-133b mimics and the miR-133b inhibitor were synthesized by Shanghai GenePharma Co., Ltd (Shanghai, China). The transfection reagent Lipofectamine 2000 was obtained from Invitrogen Life Technologies. Primers for CTGF, miR-133b and U6-snRNA were synthesized by Changsha YRBIO Co., Ltd (Changsha, China). CTGF goat polyclonal antibody was obtained from Santa Cruz Biotechnology (Dallas, TX, USA). GAPDH rabbit polyclonal antibody was obtained from Proteintech Group, Inc. (Chicago, IL, USA). The horseradish peroxidase (HRP)-goat anti-rabbit antibody and the HRP-rabbit anti-goat antibody used for western blot analysis were obtained from Zhong Shan Goldenbridge Biotechnology Co., Ltd (Beijing, China). The PCR 2.1 vector was purchased from Invitrogen Life Technologies.

Plasmid construction and transfection. The miR expression vector pYr-pri-miRNA (YRBIO Co. Ltd) was used to construct a pYr-pri-Homo sapiens (hsa)-mir-133b plasmid. Human genome DNA was used as the template. A fragment of $\sim 600 \mathrm{bp}$ containing pri-miR-133b was cloned into the pYr vector. HT-29 and SW-620 cells were seeded at $3 \times 10^{4}$ cells per well in 12-well plates and transfected the following day in Opti-MEM medium with pYr-pri-hsa-mir-133b plasmid $(80 \mathrm{nmol} / \mathrm{l}$; Shanghai GenePharma Co., Ltd.) using Lipofectamine 2000. Cells were harvested three days prior to transfection.

RNA labeling and microarray hybridization. The CRC cell lines and transfected cell lines were sent to CapitalBio (Beijing, China) for microarray hybridization. Cells were centrifuged in RNase-free tubes treated with TRIzol ${ }^{\circledR}$ reagent (Invitrogen Life Technologies) and stored at $-80^{\circ} \mathrm{C}$ prior to RNA extraction, which was performed according to the manufacturer's instructions (Invitrogen Life Technologies). The RNA was purified using the NucleoSpinH RNA clean up kit (Macherey-Nagel, Düren, Germany). RNA concentration was assessed by Nanodrop 2000 spectrophotometry (Thermo Fisher Scientific, Waltham, MA, USA). RNA quality was determined by formaldehyde denaturation electrophoresis, and only those samples showing no degradation (ratios approaching 2:1 for the $28 \mathrm{~S}$ and $18 \mathrm{~S}$ bands) were used to generate labeled targets.
Each RNA sample was hybridized to one Roche NimbleGen Porcine Genome Expression Array (Roche, Basel, Switzerland). Briefly, double-stranded cDNA was synthesized from $6 \mathrm{mg}$ total RNA using a T7-oligo (dT) primer. The cDNA was further purified and converted into cRNA using an in vitro transcription reaction. $5 \mathrm{mg}$ cRNA was reverse transcribed to cDNA, fragmented and then labeled with Cy5-dCTP and Cy3-dCTP (GE Healthcare, Little Chalfont, UK) using Taq DNA enzyme (Carolina Biosystems, Beijing, China). These labeled cDNA fragments were hybridized for $16 \mathrm{~h}$ at $45^{\circ} \mathrm{C}$ using the $2 \mathrm{X} \mathrm{GE}$ Hybridization Buffer (Roche Genome Expression Array kit; Roche). The GeneChips were then washed, stained and scanned with an Agilent G2565CA Microarray Scanner (Agilent Technologies, Santa Clara, CA, USA).

Data analysis. Agilent Feature Extraction software (version 11.0.1.1; Agilent Technologies) was used to analyze the acquired array images. Quantile normalization and subsequent data processing were conducted using the GeneSpring GX v12.0 software package (Agilent Technologies). Following quantile normalization of the raw data, genes with $\geq 1$ out of 2 samples with raw data in the microarray were selected for further data analysis. Differential expression of genes was considered significant if the false discovery rate (FDR) was calculated to the correct P-value (controlling the expected FDR to no more than 5\%) and the fold change $>11.5 \mid$. Hierarchical clustering was performed using the Agilent GeneSpring GX software (version 12.0; Agilent Technologies).

RNA extraction and reverse transcription-quantitative polymerase chain reaction ( $R T-q P C R)$. Total RNA was isolated using E.Z.N.A. ${ }^{\circledR}$ Total RNA kit II (Omega Bio-Tek Inc., Norcross, GA, USA). miRNA was isolated using E.Z.N.A. PF miRNA Isolation kit (Omega Bio-Tek Inc.). Large RNA reverse transcription was performed using the RevertAid first-strand cDNA synthesis kit (Thermo Fisher Scientific Inc., Vilnius, Lithuania). miRNA reverse transcription was performed using an All-in-One ${ }^{\mathrm{TM}}$ miRNA qRT-PCR Detection kit (GeneCopoeia, Inc., Rockville, MD, USA). qPCR was performed using Real-Time Quantitative PCR SYBR Green detection reagent (Cowin Biotech Co., Ltd., Beijing, China). miRNA qRT-PCR was performed using All-in-One ${ }^{\mathrm{TM}}$ miRNA qRT-PCR Detection kit (GeneCopoeia, Inc.). Primers utilized for cDNA amplification are summarized in Table I. The following reaction conditions were used: An initial $10 \mathrm{~min}$ at $95^{\circ} \mathrm{C}$; then 40 cycles of $20 \mathrm{sec}$ at $94^{\circ} \mathrm{C}, 15 \mathrm{sec}$ at $59^{\circ} \mathrm{C}$ and $30 \mathrm{sec}$ at $72^{\circ} \mathrm{C}$. The standard $\mathrm{PCR}$ reaction mixture then underwent a final extension for $5 \mathrm{~min}$ at $72^{\circ} \mathrm{C}$, while the quantitative PCR mixture was subjected to melting curve analysis to validate the reaction product specificity. The relative expression of CTGF was normalized to that of GAPDH using the $2^{-\Delta \Delta C T}$-method. The relative expression of miR-133b was normalized to that of U6-snRNA using the $2^{-\Delta \Delta C T}$-method. Each sample was amplified in triplicate.

Dual-luciferase reporter gene analyses. SW-620 and HT-29 cells were seeded in a 96 -well plate at $2 \times 10^{4}$ cells per well and incubated overnight. miR-133b was transfected into SW-620 and HT-29 cells using Lipofectamine 2000. At $24 \mathrm{~h}$ after transfection, cells were transfected with the pYr-MirTarget Renilla 
Table I. Primers for reverse transcription-quantitative polymerase chain reaction.

\begin{tabular}{|c|c|c|c|}
\hline Gene detected & Primer & Sequence & Product size \\
\hline CTGF & $\begin{array}{l}\text { Forward } \\
\text { Reverse }\end{array}$ & $\begin{array}{l}\text { TGGCAGGCTGATTTCTAGGT } \\
\text { GGTGCAAACATGAACTTTTGG }\end{array}$ & $191 \mathrm{bp}$ \\
\hline miR-133b & $\begin{array}{l}\text { Forward } \\
\text { Reverse }\end{array}$ & $\begin{array}{l}\text { CTTTGGTCCCCTTCAACCA } \\
\text { GTGCAGGGTCCGAGGT }\end{array}$ & 72 bp \\
\hline U6 & $\begin{array}{l}\text { Forward } \\
\text { Reverse }\end{array}$ & $\begin{array}{l}\text { CTCGCTTCGGCAGCACA } \\
\text { AACGCTTCACGAATTTGCGT }\end{array}$ & $94 \mathrm{bp}$ \\
\hline GAPDH & $\begin{array}{l}\text { Forward } \\
\text { Reverse }\end{array}$ & $\begin{array}{l}\text { GAAGGTGAAGGTCGGAGT } \\
\text { CATGGGTGGAATCATATTGGAA }\end{array}$ & $155 \mathrm{bp}$ \\
\hline
\end{tabular}

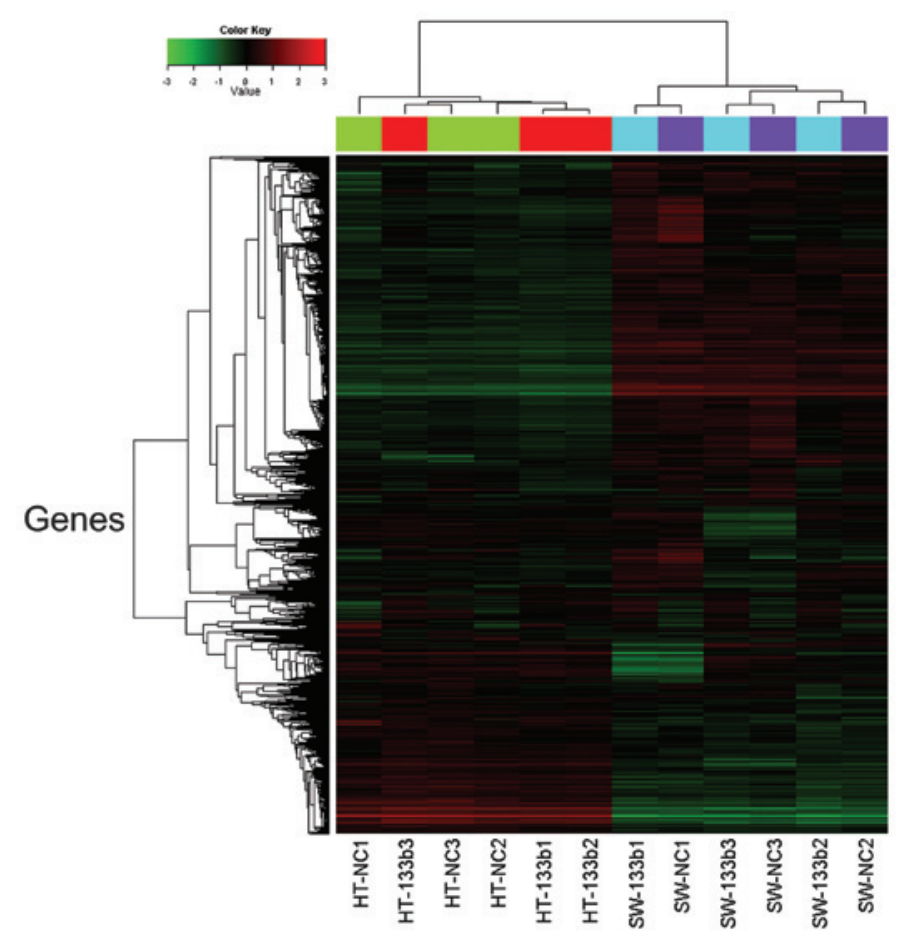

Figure 1. Hierarchical cluster analysis of genes showed the differential expression of genes between miR-133b-transfected HT-29 and SW-620 cells and untransfected cells. Horizontal lines represent single genes, and each column represents a single sample. Red indicates upregulated genes, whereas green indicates downregulated genes. Gene upregulation was defined as a fold change in relative transcription levels of Log FC $>1.5$ and FDR $<0.05$. Downregulation was defined as Log FC $<-1.5$ and FDR $<0.05$. Genes with relative transcription levels of $-1.5<$ Log FC $<1.5$ were considered to show no significant change. miR-133b, microRNA-133b; Log FC, Log fold change; FDR, false discovery rate.

luciferase plasmid in combination with the CTGF 3'-UTR expression plasmid (Cignal Reporter Assay kit; Qiagen, Hilden, Germany). Cells were harvested at $48 \mathrm{~h}$ following transfection and assayed for luciferase activity using the Dual-Luciferase Reporter Assay System (Promega Corp., Madison, WI, USA). Luciferase activity was normalized against that of Renilla luciferase. Luminescence was quantified with a luminometer (Sirius single tube luminometer, Belthold, Wildbad, Germany). All experiments were performed in triplicate.

Tissue specimens. The human CRC tissues (histologically confirmed as adenocarcinoma of the colon) and adjacent non-cancerous tissue counterparts used for RT-qPCR and western blotting were obtained from patients who underwent surgical resection of CRC at the Third Affiliated Hospital of Central South University (Hunan, China) once written informed consent had been obtained. All specimens were snap frozen in liquid nitrogen for $24 \mathrm{~h}$ and then stored at $-80^{\circ} \mathrm{C}$ prior to further processing. The study was approved by the ethics committee of the Third XiangYa Hospital of Central South University (Hunan, China). Patient consent was obtained from the patient as well as the patient's family.

Western blot analysis. Total protein from tissues samples and the cultured cell lines was harvested in a radioimmunoprecipitation assay lysis buffer (Sigma-Aldrich Co, St. Louis, MO, USA). A volume of extract equivalent to $100 \mu \mathrm{g}$ total protein was separated by 7.5\% SDS-PAGE (Well Biological Co., Ltd, Changsha, China) and transferred onto polyvinylidene difluoride membranes (Invitrogen Life Technologies). The membranes 
Table II. List of top 20 differentially expressed genes in miR-133b-transfected HT-29 and SW-620 cells.

\begin{tabular}{lccr}
\hline Accession number & Gene symbol & Fold change & Regulation \\
\hline NM_019899 & ABCC1 & 6.0329833 & Downregulated \\
NM_012244 & SLC7A8 & 4.2845231 & Downregulated \\
NM_004156 & PPP2CB & 4.0159174 & Downregulated \\
NM_001664 & RhoA & 3.6389074 & Downregulated \\
NM_004994 & MMP9 & 3.3274931 & Downregulated \\
NM_022075 & CERS2 & 3.2985491 & Downregulated \\
NM_144649 & TMEM71 & 2.8322382 & Downregulated \\
NM_000627 & LTBP1 & 2.7411814 & Downregulated \\
NM_022068 & PIEZO2 & 2.7360535 & Downregulated \\
NM_001901 & CTGF & 2.6958947 & Downregulated \\
NM_182485 & CPEB2 & 2.5642369 & Downregulated \\
NM_003706 & PLA2G4C & 2.5298514 & Downregulated \\
NM_030762 & BHLHE41 & 2.4895813 & Downregulated \\
NM_001771 & CD22 & 2.3959713 & Downregulated \\
NM_001430 & EPAS1 & 2.3748767 & Downregulated \\
NM_003948 & CDKL2 & 2.3225446 & Downregulated \\
NM_007129 & ZIC2 & 2.3078060 & Downregulated \\
NM_006398 & UBD & 2.2501510 & Downregulated \\
NM_001018077 & NR3C1 & 2.1797345 & Downregulated \\
NM_001266 & CES1 & 2.1734946 & Downregulated \\
\hline
\end{tabular}

were incubated with Tris-buffered saline and Tween-20 (TBST) (Well Biological Co., Ltd) containing 5\% skimmed milk at $4^{\circ} \mathrm{C}$ overnight. Membranes were then incubated with the following primary antibodies: goat anti-CTGF polyclonal antibody (1:500) and GAPDH rabbit polyclonal antibody (1:2,000), at room temperature for $2 \mathrm{~h}$. The membranes were then washed three times with TBST and incubated with HRP-conjugated rabbit anti-goat antibody $(1: 10,000)$ and HRP-conjugated goat anti-rabbit antibody $(1: 2,000)$ for $1 \mathrm{~h}$. Membranes were then washed three times with TBST and treated with western blotting luminal reagent (Pierce ECL Western Blotting kit; Thermo Fisher Scientific Inc., Vilnius, Lithuania) for band visualization, the results were obtained using an ImageQuant LAS 350 (GE Healthcare) and quantified by densitometry. Blots were performed in triplicate.

Statistical analysis. All statistical analyses were conducted using the SPSS version 16.0 software package (SPSS, Inc., Chicago, IL, USA). Data are expressed as the mean \pm standard deviation. Differences between groups were compared using analysis of variance and two-tailed t-tests. The $\chi^{2}$ test was used to analyze the significance of the association between miR-133b expression and clinicopathological characteristics. Each experiment was performed independently at least twice and obtained similar results. $\mathrm{P}<0.05$ was considered to indicate a statistically significant difference.

\section{Results}

Microarray analysis of CRC cell lines. In order to investigate whether there was an effect of miR-133b on the expression of individual genes, cluster analysis was performed to organize genes into clusters based on the similarities of their expression levels. Statistical analysis using an unpaired t-test demonstrated that a total of 103 genes were differentially expressed (72 upregulated and 31 downregulated) between HT-29 and SW-620 cells (Fig. 1). The 20 genes downregulated to the greatest extent were differentially expressed in miR-133b-transfected HT-29 and SW-620 cells, compared with the corresponding type of untransfected cells, with the fold change calculated based on the mean value of the two cell groups (Table II). CTGF was identified by microarray analysis as a potential target candidate of miR-133b interaction $(\log \mathrm{FC}=-4.41, \mathrm{FDR}<0.05)$ and was selected for further study.

$C T G F$ is a direct target of miR-133b. As miRNAs have been implicated in the repression of gene expression, it was hypothesized that miR-133b may target a negative regulator of the expression of CTGF. Using computational screening (TargetScan; Whitehead institute for Biomedical Research, www.targetscan.org), it was shown that the 3'UTR of CTGF contains a potential binding site for miR-133b (Fig. 2A). The transfection efficiency of synthetic miR-133b was evaluated in the human HT-29 and SW-620 CRC cell lines. As shown in Fig. 2B, RT-qPCR analysis demonstrated that miR-133b levels in miR-133b-transfected cells were significantly higher than those in untransfected or control-transfected cells (HT-29 cells, $\mathrm{P}<0.01$; SW-620 cells, $\mathrm{P}<0.01$ ).

In order to investigate whether miR-133b expression influenced CTGF expression, mRNA and protein levels of CTGF were examined in miR-133b-transfected cell 

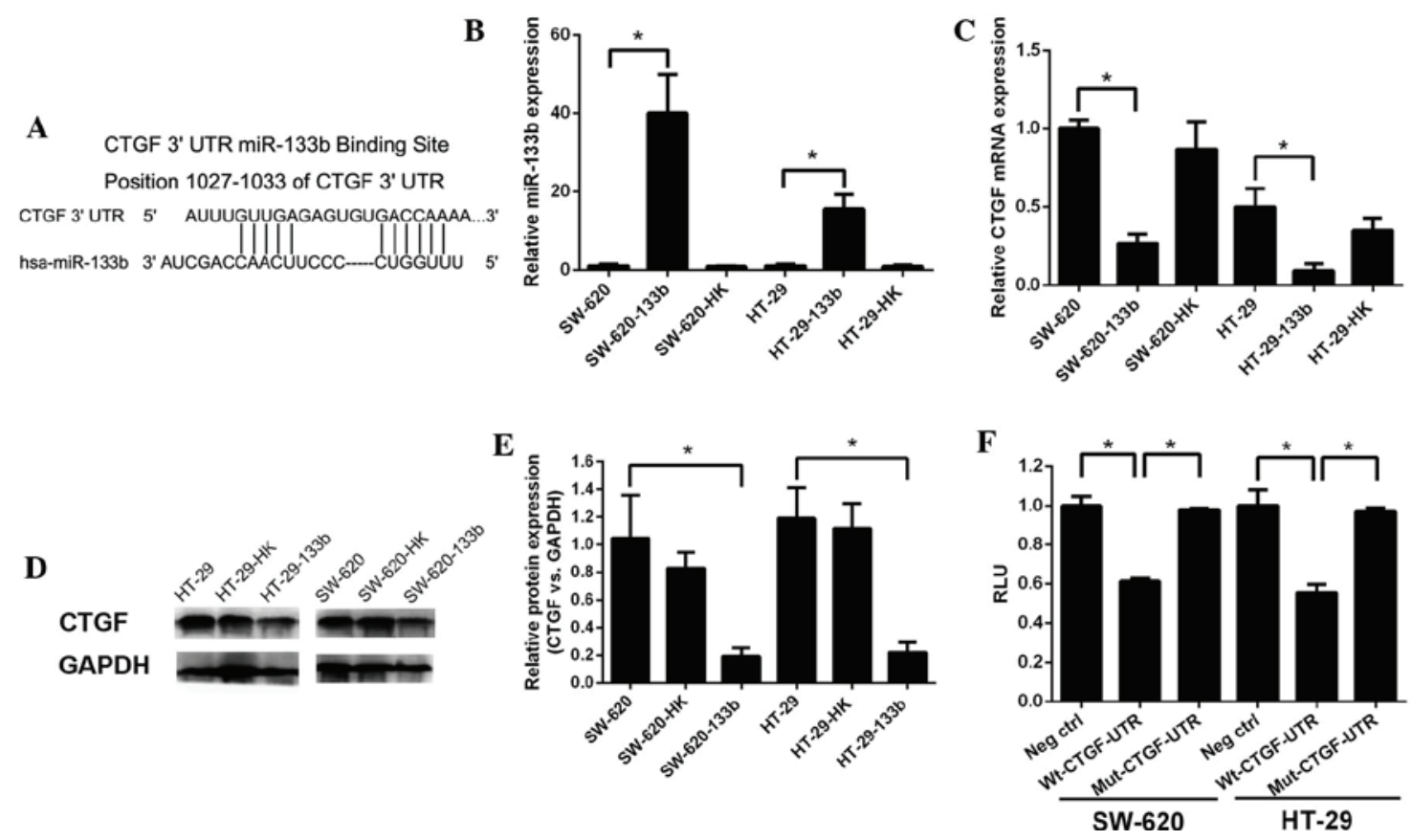

Figure 2. Posttranscriptional repression of CTGF expression by miR-133b. (A) Position of the miR-133b target site along the 3'-UTR of CTGF predicted by miRBase targets. (B) Endogenous miR-133b levels were assessed by RT-qPCR. (C) Endogenous CTGF levels were confirmed by RT-qPCR. (D) Protein expression of CTGF was determined by western blot analysis. (E) CTGF protein levels are expressed as a percentage relative to GAPDH in HT-29 and SW-620 cells. Following transfection with the miR-133b expressing vector, HT-29 and SW-620 cells exhibited a significant decrease in CTGF expression. (F) Luciferase assay. SW-620 and HT-29 cells were cotransfected with miR-133b vectors together with a firefly luciferase vector, containing CTGF 3'-UTR or mutant CTGF 3'-UTR, and a Renilla luciferase control. Histogram indicates the RLU in different transfected cells. All experiments were repeated three times. Columns and bars represent the mean and standard deviation, respectively. "P<0.05. CTGF, connective tissue growth factor; miR-133b, microRNA-133b; RT-qPCR, reverse transcription-quantitative polymerase chain reaction; RLU, relative luciferase unit; Wt, wild-type; Mut, mutated; hsa, Homo sapiens.

lines (Fig. 2C-E). miR-133b transfection significantly reduced the levels of CTGF mRNA compared with those in untransfected or control-transfected cells (SW-620 cells, $\mathrm{P}<0.01$; HT-29 cells, $\mathrm{P}<0.05$; Fig. $2 \mathrm{C}$ ). Western blot analysis showed that the protein levels of CTGF were also downregulated following overexpression of miR-133b in SW-620 and HT-29 cells, compared with those in the controls (Fig. 2D). Quantification of protein bands revealed a four-fold decrease in CTGF protein following overexpression of miR-133b ( $\mathrm{P}<0.05$; Fig. 2E).

In order to determine whether the observed reduction in CTGF expression is directly driven by miR-133b, reporter gene assays were conducted in HT-29 and SW-620 cells. The entire 3'UTR of CTGF was cloned into the pYr-MirTarget-control vector, creating a luciferase reporter gene containing the seed match for miR-133b. In addition, a mutated reporter construct was generated, in which the miR-133b seed match sequence was deleted from the 3'UTR of CTGF. Co-transfection with the CTGF wild-type-3'UTR construct and miR-133b yielded a significantly reduced relative luciferase activity (SW-620 cells, P $<0.05$; HT-29 cells, $\mathrm{P}<0.05$; Fig. 2F). However, the luciferase activity of the reporter construct that had been mutated at the specific miR-133b target site was not reduced in comparison with that in untransfected cells, when simultaneously transfected with miR-133b (SW-620 cells, $\mathrm{P}<0.05$; HT-29 cells, $\mathrm{P}<0.05$; Fig. 2F). This suggested that the 3 '-UTR of CTGF is a functional target site for miR-133b in HT-29 and SW-620 cancer cells.
Verification that CTGF expression is inversely correlated with miR-133b expression in human CRC specimens. In order to gain insight into the biological functions of miR-133b and CTGF in human colorectal carcinogenesis, the expression of miR-133b in human CRC tissues and paired healthy counterparts was analyzed using RT-qPCR. Following this, the expression levels of miR-133b and CTGF mRNA were measured using comparisons with the human CCD-18Co healthy colon cell line as a reference. The relative expression levels of miR-133b in tumor specimens compared with those in the CCD-18Co cells $(0.55 \pm 0.41)$ were significantly lower than the relative expression levels in non-tumor specimens compared with those in CCD-18Co cells $(1.32 \pm 0.24 ; \mathrm{P}<0.001)$. The relative expression levels of CTGF mRNA in tumor specimens $(1.46 \pm 0.36)$ were significantly higher than those $(0.57 \pm 0.32)$ in the corresponding non-tumor specimens $(\mathrm{P}<0.001$; Fig. 3A).

The effect of miR-133b expression on that of CTGF was assessed using data obtained from RT-qPCR. A significant negative correlation was observed between CTGF mRNA and miR-133b expression ( $\mathrm{n}=71, \mathrm{r}=-0.385, \mathrm{P}<0.01$, Pearson's correlation; Fig. 3B). These data demonstrated a reciprocal regulation of miR-133b and CTGF, suggesting that downregulation of miR-133b may lead to CTGF overexpression in CRC tissues.

Furthermore, western blot analysis was conducted in order to determine whether protein expression of CTGF was consistent with mRNA expression. As hypothesized, the alterations in CTGF protein levels in tumor samples compared with those in non-tumor samples were in accordance with changes in 
A

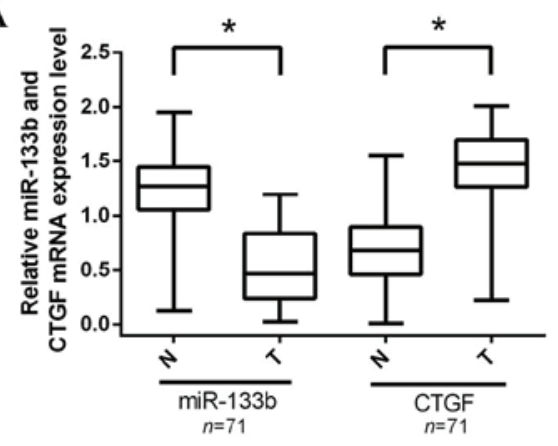

C

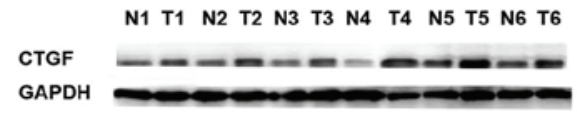

B

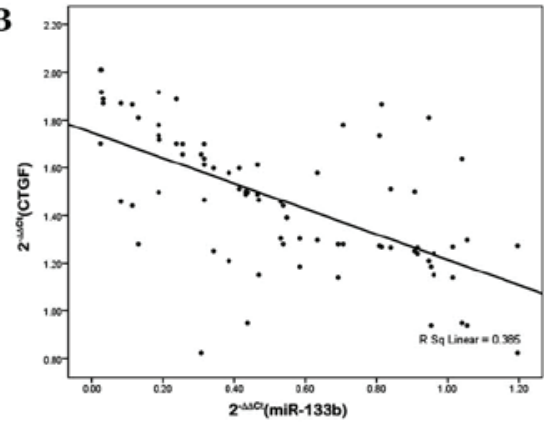

D

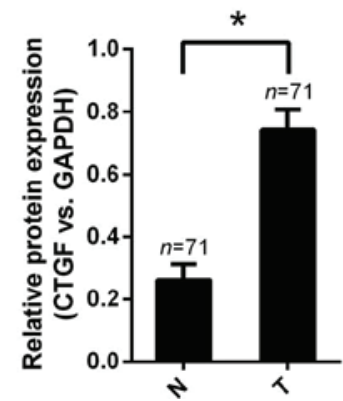

Figure 3. Expression of miR-133b and CTGF in CRC tissues. (A) Expression of miR-133b was measured using 71 surgical specimens of human CRC tissues and adjacent normal tissues for reverse transcription quantitative polymerase chain reaction. The correction values of CTGF and miR-133b expression were calculated by dividing the CTGF and miR-133b values by the endogenous reference values (GAPDH and U6) concurrently examined in each sample. Boxes indicate the first quartile, median and third quartile values of CTGF/GAPDH and miR-133b/U6; bars indicate the minimum and maximum values recorded (B) miR-133b and CTGF expression levels were inversely correlated. $2^{-\Delta \Delta C t}$ values of miR-133b and CTGF mRNA were subjected to a Pearson correlation analysis $(\mathrm{r}=-0.385, \mathrm{P}<0.01)(\mathrm{C})$ Western blot analysis of CTGF in human CRC tissues. (D) CTGF protein expression in tumor compared with normal tissues relative to GAPDH. All experiments were repeated three times. Columns and bars represent the mean and standard deviation, respectively. "P<0.05. miR-133b, microRNA-133b; CTGF, connective tissue growth factor; CRC, colorectal cancer; N, non-tumor; T, tumor.

mRNA levels in these groups. Protein levels of CTGF were verified in $\mathrm{CRC}$ tissues $(\mathrm{P}<0.05$; Fig. $3 \mathrm{C}$ and $\mathrm{D})$.

Correlations between miR-133b, CTGF and clinicopathological features. In order to evaluate the correlation between miR-133b and CTGF mRNA expression as well as clinicopathological factors relevant to CRC, the samples were divided into groups according to whether they exhibited low or high expression using the median values of miR-133b and CTGF mRNA levels. Patients with expression levels of miR-133b in tumor tissues below the median value of 0.47 were assigned to the low expression group $(n=36)$, while patients with miR-133b expression levels in tumor tissue $\geq 0.47$ were assigned to the high expression group $(n=35)$. In addition, patients with tumor CTGF mRNA expression levels below the median value of 1.49 were assigned to the low expression group $(n=36)$ and patients with tumor CTGF mRNA expression levels $\geq 1.49$ were assigned to the high expression group $(\mathrm{n}=35)$. Current staging for colorectal cancer is based on the 7th edition of AJCC Cancer Staging Manual (16). There was a significant difference between the groups with low and high expression of miR-133b in terms of cell differentiation, lymph node metastasis and clinical stage, with a low expression being associated with poor differentiation, lymph node metastasis and a more advanced stage $(\mathrm{P}<0.05$; Table III). However, no significant difference was found between the groups with low and high expression of miR-133b in terms of patient age, gender, tumor site or extramural vascular invasion status $(\mathrm{P}>0.05)$.
Furthermore, a high expression of CTGF was associated with advanced clinical stage (stage III or IV vs. I or II) and lymph node metastasis $(\mathrm{P}<0.05$; Table III). No significant difference was detected between the groups with low or high expression of CTGF mRNA in terms of patient age, gender, tumor site, tumor differentiation or extramural vascular invasion status $(\mathrm{P}>0.05)$. The association between the levels of miR-133b and CTGF mRNA expression and the clinicopathological characteristics is summarized in Table III. In brief, the data suggest that miR-133b and CTGF may be candidate therapeutic targets in colorectal cancer, in particular in stage III and stage IV tumors.

\section{Discussion}

Previous studies have indicated that miR-133b is differentially expressed in CRC tissue samples and cell lines compared with healthy tissues $(6,17,18)$. The present study demonstrated that CTGF is a potential functional target of miR-133b. The expression levels of miR-133b and CTGF were shown to be correlated with the clinical stage and lymph node metastasis in colorectal cancer tissues, indicating that levels of miR-133b and CTGF may be useful as clinical biomarkers of tumor malignancy and thus prognosis.

Downregulation of miR-133b has been reported in a number of cancer types, including gastric, lung and bladder cancers (19-21). The high frequency of miR-133b downregulation in various types of cancer suggests that miR-133b may be involved in oncogenesis. In the present study, data analysis confirmed that specimens with a low miR-133b were more 
Table III. Association of clinical and pathological characteristics with high or low miR-133b and CTGF mRNA expression.

\begin{tabular}{|c|c|c|c|c|c|c|c|}
\hline \multirow[b]{2}{*}{$\begin{array}{l}\text { Clinicopathological } \\
\text { features }\end{array}$} & \multirow[b]{2}{*}{ Number } & \multicolumn{3}{|c|}{ miR-133b } & \multicolumn{3}{|c|}{ CTGF } \\
\hline & & $\begin{array}{c}\text { Low }^{\mathrm{a}} \\
(\mathrm{n}=36)\end{array}$ & $\begin{array}{l}\operatorname{High}^{\mathrm{a}} \\
(\mathrm{n}=35)\end{array}$ & P-value & $\begin{array}{l}\text { Low }^{\mathrm{a}} \\
(\mathrm{n}=36)\end{array}$ & $\begin{array}{l}\text { High }^{\mathrm{a}} \\
(\mathrm{n}=35)\end{array}$ & P-value \\
\hline Age (years) & 71 & 58.8 & 61.2 & 0.687 & 60.1 & 59.9 & 0.733 \\
\hline Gender (male:female) & $(38: 33)$ & $(21: 15)$ & $(17: 18)$ & 0.410 & $(16: 20)$ & $(22: 13)$ & 0.120 \\
\hline \multicolumn{8}{|l|}{ Tumor site $^{\mathrm{b}}$} \\
\hline Right & 23 & 12 & 11 & 0.347 & 10 & 13 & 0.677 \\
\hline Left & 23 & 14 & 9 & & 12 & 11 & \\
\hline Rectum & 25 & 10 & 15 & & 14 & 11 & \\
\hline \multicolumn{8}{|l|}{ Tumor differentiation } \\
\hline Well & 2 & 0 & 2 & $0.011^{\mathrm{c}}$ & 1 & 1 & 0.943 \\
\hline Moderate & 48 & 20 & 28 & & 25 & 23 & \\
\hline Poor & 21 & 16 & 5 & & 10 & 11 & \\
\hline \multicolumn{8}{|l|}{ Staging } \\
\hline I & 6 & 1 & 5 & 0.263 & 4 & 2 & 0.084 \\
\hline II & 18 & 7 & 11 & & 13 & 5 & \\
\hline III & 33 & 18 & 15 & & 12 & 21 & \\
\hline IV & 14 & 10 & 4 & & 7 & 7 & \\
\hline (III, IV : I, II) & $(47: 24)$ & $(28: 8)$ & $(19: 16)$ & $0.036^{\mathrm{c}}$ & $(19: 17)$ & $(28: 7)$ & $0.015^{\mathrm{c}}$ \\
\hline \multicolumn{8}{|l|}{ Lymph node } \\
\hline N0 & 29 & 8 & 21 & $0.037^{\mathrm{c}}$ & 20 & 9 & $0.034^{\mathrm{c}}$ \\
\hline $\mathrm{N} 1$ & 23 & 13 & 10 & & 8 & 15 & \\
\hline $\mathrm{N} 2$ & 19 & 15 & 4 & & 8 & 11 & \\
\hline \multicolumn{8}{|c|}{ Extramural vascular invasion } \\
\hline Present & 14 & 10 & 4 & 0.083 & 8 & 6 & 0.591 \\
\hline Absent & 57 & 26 & 31 & & 28 & 29 & \\
\hline
\end{tabular}

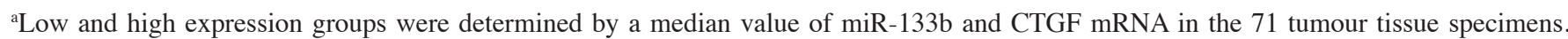
${ }^{b}$ Right, cecum to splenic flexture; left, splenic flexture to sigmoid colon. ${ }^{c}$ Statistical significance $(\mathrm{P}<0.05)$. miR-133b, microRNA-133b; CTGF, connective tissue growth factor.

likely to exhibit poor cell differentiation, lymph node metastasis and advanced clinical stage. No significant association was detected between the expression levels of miR-133b and the patient age or gender, the tumor site or the extramural vascular invasion status in 71 patients with CRC.

The CTGF protein is a $38 \mathrm{kDa}$ extracellular matrix protein, which is a member of the $\mathrm{CCN}$ cysteine-rich family of proteins. CTGF has multiple functions. It interacts with integrin receptors and numerous growth factors. In addition, it serves as a biostore for angiogenic factors such as the vascular endothelial growth factor (22). CTGF modulates certain cellular processes under normal or pathological conditions, including cell adhesion, migration, proliferation, chemotaxis, apoptosis, extracellular matrix (ECM) deposition and angiogenesis (23). TGF- $\beta$ and its downstream mediators, including CTGF, are initiators of invasion and migration processes in cancer progression (24). These processes may be independent from each other, since the TGF- $\beta$ and CTGF-dependent induction of epithelial to mesenchymal transition was shown to be independent of the profibrotic effects of TGF- $\beta$ in proximal tubular cells (25). CTGF has been implicated in other processes necessitating cell migration, such as the re-epithelialization of the cornea, which is mediated through TGF- $\beta$ and CTGF signaling, leading to downstream extracellular signal-regulated kinase $1 / 2$ and p38 mitogen-activated protein kinase activation (25). CTGF stimulates integrin expression and has been shown to bind directly to endothelial cell surface integrins $(27,28)$, thereby mediating ECM interaction and prolonging cell survival. CTGF is known to mediate anchorage-independent cancer cell growth. Evidence for this is based on findings in which anti-CTGF treatment has been shown to inhibit anchorage-independent growth in vitro, primary tumor growth in vivo and the development of macroscopic lymph node metastases (29).

The present study demonstrated that miR-133b targets CTGF, indicating a potential mechanism associated with colorectal tumorigenesis. In the luciferase reporter assay, wild-type CTGF was shown to be specifically responsive to miR-133b overexpression. By contrast, the mutated CTGF, which contained a mutation in the miR-133b binding sites within the CTGF 3'UTR, successfully abolished the effect of miR-133b transfection on luciferase activity. This was 
confirmed using RT-qPCR in CRC specimens, which showed that miR-133b expression was negatively correlated with CTGF expression. RT-qPCR and western blot analyses demonstrated that CTGF was highly expressed in human CRC cell lines, as well as in primary tumors from patients with CRC. Furthermore, a significant correlation was observed between increased levels of CTGF and advanced tumor, nodes and metastasis stage. These findings indicated that miR-133b may lead to lymph node metastasis and advanced clinical stage via regulation of CTGF.

In conclusion, the present study demonstrated that CTGF was a target gene of miR-133b in the human colorectum. The role of miR-133b in CRC was further highlighted by the demonstration of a correlation between its expression and certain clinicopathological characteristics. These results suggested that CTGF may be used to establish a possible link between the biological function of miR-133b and the pathogenesis of CRC. Furthermore, miR-133b and CTGF may be candidate therapeutic targets in colorectal cancer.

\section{Acknowledgements}

This study was supported by the Planned Science and Technology Project of Hunan Province, China (grant no. 2008FJ3160) and the Chinese National Science Foundation (grant no. 81172298).

\section{References}

1. Rossi S, Di Narzo AF, Mestdagh P, et al: microRNAs in colon cancer: a roadmap for discovery. FEBS Lett 586: 3000-3007, 2012.

2. Gansler T, Ganz PA, Grant M, et al: Sixty years of CA: a cancer journal for clinicians. CA Cancer J Clin 60: 345-350, 2010

3. Lamy P, Andersen CL, Dyrskjøt L, Tørring N, Ørntoft T and Wiuf C: Are microRNAs located in genomic regions associated with cancer? Br J Cancer 95: 1415-1418, 2006.

4. Duisters RF, Tijsen AJ, Schroen B, et al: miR-133 and miR-30 regulate connective tissue growth factor: implications for a role of microRNAs in myocardial matrix remodeling. Circ Res 104: 170-178, 6p, 2009.

5. Wen D, Li S, Ji F, et al: miR-133b acts as a tumor suppressor and negatively regulates FGFR1 in gastric cancer. Tumour Biol 34 793-803, 2013.

6. Hu G, Chen D, Li X, Yang K, Wang H and Wu W: miR-133b regulates the MET proto-oncogene and inhibits the growth of colorectal cancer cells in vitro and in vivo. Cancer Biol Ther 10 : 190-197, 2010

7. Chen CC and Lau LF: Functions and mechanisms of action of CCN matricellular proteins. Int J Biochem Cell Biol 41: 771-783, 2009.

8. Chu CY, Chang CC, Prakash E and Kuo ML: Connective tissue growth factor (CTGF) and cancer progression. J Biomed Sci 15: 675-685, 2008.

9. Garcia P, Leal P, Ili C, Brebi P, Alvarez H and Roa JC: Inhibition of connective tissue growth factor $(\mathrm{CTGF} / \mathrm{CCN} 2)$ in gallbladder cancer cells leads to decreased growth in vitro. Int J Exp Pathol 94: 195-202, 2013

10. Kang Y, Siegel PM, Shu W, et al: A multigenic program mediating breast cancer metastasis to bone. Cancer Cell 3: 537-549, 2003.
11. Deng YZ, Chen PP, Wang Y, et al: Connective tissue growth factor is overexpressed in esophageal squamous cell carcinoma and promotes tumorigenicity through beta-catenin-T-cell factor/Lef signaling. J Biol Chem 282: 36571-36581, 2007.

12. Wenger C, Ellenrieder V, Alber B, et al: Expression and differential regulation of connective tissue growth factor in pancreatic cancer cells. Oncogene 18: 1073-1080, 1999.

13. Kubo M, Kikuchi K, Nashiro K, et al: Expression of fibrogenic cytokines in desmoplastic malignant melanoma. Br J Dermatol 139: 192-197, 1998.

14. Shakunaga T, Ozaki T, Ohara N, et al: Expression of connective tissue growth factor in cartilaginous tumors. Cancer 89: 1466-1473, 2000.

15. Lee HK, Bier A, Cazacu S, et al: MicroRNA-145 is downregulated in glial tumors and regulates glioma cell migration by targeting connective tissue growth factor. PLoS One 8: e54652, 2013.

16. Edge S, Byrd DR, Compton CC, Fritz AG, Greene FL and Trotti A (eds): AJCC Cancer Staging Manual. 7th edition. Springer, New York, NY, 2010.

17. Xiang KM and Li XR: MiR-133b acts as a tumor suppressor and negatively regulates TBPL1 in colorectal cancer cells. Asian Pac J Cancer Prev 15: 3767-3772, 2014.

18. Lin CW, Li XR, Zhang Y, et al: TAp63 suppress metastasis via miR-133b in colon cancer cells. Br J Cancer. 110: 2310-2320, 2014.

19. Wen D, Li S, Ji F, Cao H, Jiang W, Zhu J and Fang X: Mir-133b acts as a tumor suppressor and negatively regulates FGFR1 in gastric cancer. Tumour Biol 34: 793-803, 2013.

20. Crawford M, Batte K, Yu L, Wu X, Nuovo GJ, Marsh CB, Otterson GA and Nana-Sinkam SP: MicroRNA-133b targets pro-survival molecules MCL-1 and BCL2L2 in lung cancer. Biochem Biophys Res Commun 388: 483-489, 2009.

21. Ichimi T, Enokida H, Okuno Y, Kunimoto R, Chiyomaru T, Kawamoto K, Kawahara K, Toki K, Kawakami K, Nishiyama K, et al: Identification of novel microRNA targets based on microRNA signatures in bladder cancer. Int J Cancer 125: 345-352, 2009.

22. Duisters RF, Tijsen AJ, Schroen B, et al: miR-133 and miR-30 regulate connective tissue growth factor: implications for a role of microRNAs in myocardial matrix remodeling. Circ Res 104: 170-178, 2009.

23. Hofmeister V, Schrama D and Becker JC: Anti-cancer therapies targeting the tumor stroma. Cancer Immunol Immunother 57: 1-17, 2008.

24. Wendt MK, Smith JA and Schiemann WP: Transforming growth factor- $\beta$-induced epithelial-mesenchymal transition facilitates epidermal growth factor-dependent breast cancer progression. Oncogene 29: 6485-6498, 2010.

25. Wang B, Herman-Edelstein M, Koh P, Burns W, Jandeleit-Dahm K, Watson A, Saleem M, Goodall GJ, Twigg SM, Cooper ME and Kantharidis P: E-cadherin expression is regulated by miR-192/215 by a mechanism that is independent of the profibrotic effects of transforming growth factor-beta. Diabetes 59: 1794-1802, 2010.

26. Secker GA, Shortt AJ, Sampson E, Schwarz QP, Schultz GS and Daniels JT: TGFbeta stimulated re-epithelialisation is regulated by CTGF and Ras/MEK/ERK signalling. Exp Cell Res 314: 131-142, 2008.

27. Babic AM, Chen CC and Lau LF: Fisp12/mouse connective tissue growth factor mediates endothelial cell adhesion and migration through integrin alphavbeta3, promotes endothelial cell survival, and induces angiogenesis in vivo. Mol Cell Bio 19: 2958-2966, 1999.

28. Lau LF and Lam SC: The CCN family of angiogenic regulators: the integrin connection. Exp Cell Res 248: 44-57, 1999.

29. Dornhöfer N, Spong S, Bennewith K, Salim A, Klaus S, Kambham N, Wong C, Kaper F, Sutphin P, Nacamuli R, et al: Connective tissue growth factor-specific monoclonal antibody therapy inhibits pancreatic tumor growth and metastasis. Cancer Res 66: 5816-5827, 2006. 